\title{
Automatic Recognition and Construction of Draft Angle for Injection Mold Design
}

\author{
Wen-Ren Jong, Tai-Chih Li, Yu-Wei Chen, Yu-Hung Ting \\ Department of Mechanical Engineering and R \& D Center for Mold and Molding Technology, Chung Yuan Christian University, \\ Taiwan \\ Email: wenren@cycu.edu.tw
}

How to cite this paper: Jong, W.-R., Li, T.-C., Chen, Y.-W. and Ting, Y.-H. (2017) Automatic Recognition and Construction of Draft Angle for Injection Mold Design. Journal of Software Engineering and Applications, 10, 78-93.

http://dx.doi.org/10.4236/jsea.2017.101005

Received: November 8, 2016

Accepted: January 21, 2017

Published: January 24, 2017

Copyright $\odot 2017$ by authors and Scientific Research Publishing Inc. This work is licensed under the Creative Commons Attribution International License (CC BY 4.0). http://creativecommons.org/licenses/by/4.0/

\begin{abstract}
In the injection molding process, plastic products are difficult to demold due to friction force between the cavity and products, thus, finished products might be deformed or damaged. Therefore, designers should add a draft angle to the geometric surface of products, which is parallel to the unloading direction, in order to help the products eject smoothly from the cavity. This study uses CAD software as the main architecture to develop the function of automatic draft angle recognition and construction. The study is divided into three stages. First, the geometric features of products are identified in the CAD model by induced algorithm, then the quilts to be added in the draft design are determined and classified. Finally, draft angles are created in different ways according to different surfaces. An algorithm suitable for automatic draft recognition and construction, as well as the constraints of automatic creation of draft angle, is proposed. The feature recognition algorithm of this study can automatically inspect $90 \%$ of the surfaces to be drafted, and the automatic creation of draft features can economize $80 \%$ of required mouse clicks, thus, effectively increasing draft angle design efficiency, and preventing errors in mold design and manufacturing.
\end{abstract}

\section{Keywords}

Injection Mold Design, Draft Features Design, Feature Recognition, Automatic Design, DFX

\section{Introduction}

Under the pressure of reducing manufacturing cost and upgrading production quality in the manufacturing industry, enterprises' focus on products has diverted from "manufacture" to "design", resulting in DFX (Design for X), meaning the concept of "design considering X" [1], where X contains many aspects, 
including Assembly, Manufacturability, and Environment. DFM is most frequently used, which design concept considers manufacturing, integrates "design" and "manufacture" thoughts, and mass production is considered in product design. The shrinkage of products during injection molding is considered in product design. Therefore, products should be designed with uniform thickness in order to reduce design costs and increase mass production yield.

As mold design and molding processes will influence the final injection products, which cover a wide range of technology, it is difficult to find well-experienced talents. Moreover, if enterprises only invest in hardware equipment, production profits will be very limited. Therefore, the rich expertise from current and previous experienced workers is accumulated and inherited, academically converted into knowledge-guided design operations, and skill and knowledge are systemized and effectively applied, in order to enhance the enterprise's overall engineering capability and maximize their problem-solving ability.

In recent years, with the development of CAD/CAM technology and AI (Artificial Intelligence) technology, there are many subjects on combining product analysis and design with CAD software, including the mold design modules in commercial CAD software, such as Pro/Mold Design (PTC), IMOLD (SolidWorks), etc., providing universal mold automation functions assisting engineers to complete design works. Regarding product demolding design, Tokuyama et al. [2] used the B-Spline curve and surface to determine the draft effect of a free-form surface. Yan et al. [3] proposed making a draft angle on a composite surface, which was added to the composite surface with a fillet by the translation of a surface control point. Schreve [4] used surface control points independent of each other to create a draft angle algorithm of a B-Spline surface, which was applicable to convex surfaces. Zhou et al. [5] changed surface control points to the minimum extent to create the draft angle for B-Spline and NURBS surfaces. Nee et al. [6] and Fu et al. [7] proposed an efficient method to identify and extract undercuts in order to generate the ideal parting direction, and used the quantity of undercuts and corresponding volume to generate the ideal parting direction. Weinstein et al. [8] divided the surfaces into forward and backward directions of concave and convex surfaces, in order to determine the optimum draft angle and parting line position. Mercado-Colmenero et al. [9] poses a hybrid analysis of the discrete mesh of the plastic part, analyzing the demoldability of both the mesh nodes and the facets. The algorithm detects not only undercuts resolvable by side cores, but also non-demoldable areas, allowing the designer to modify the design in the early stages.

Based on the application of a feature recognition algorithm, many complex tasks can be operated by synthesized automation on computer. Singh et al. [10] used the rule-based approach to automatically recognize the undercut features of complicated casting die and classify the parting direction, providing designers with appropriate suggestions. Vandenbrande et al. [11] [12] found that, if there was interference between the machined features, the use of a volumetric decomposition approach to search for features was not feasible, and proposed the 
hint-based approach to solve feature interference. Verma et al. [13] proposed the hint-based machining feature recognition system, which uses the potential hint of a feature trace to recognize the machining features. Babic et al. [14] reviewed multiple methods regarding solving the automatic feature recognition problem, and discussed the constraints and future applicability of these methods. Li et al. [15] proposed an integrated approach based on Feature Hints, the Graph Theory, and an Artificial Neural Network, which could recognize overlapped machining features. Lai et al. [16] proposed a virtual loop recognition method for detecting all types of loops used in CAD models, and for enabling the recognition of features across multiple faces.

In terms of the integration of knowledge and design, Turng et al. [17] developed a plastic injection mold knowledge management system, and built it in a network, using information technology to overcome geographic location and time barriers to save in-house knowledge. The system divides knowledge into five subjects, including design, material, process, CAE, and problem removal. The operation interface enables the user to easily obtain data. Fuh et al. [18] proposed a three-dimensional mold design system (IMOLD) for business CAD software. This system divides modeling into four major stages, the creation of a project, cavity and core creation, runner design, and mold base design, which provides the functions to control the project and assists the designer to complete the project correctly. Chan et al. [19] proposed an interactive knowledge-based mold design system (IKB-MOULD), including mold design processes and injection mold knowledge management. An interactive operating environment was provided by $\mathrm{CAD}$, which accelerates injection mold design processing, and renders design standardization easier. Jong et al. [20] [21] [22] planned and created a knowledge-based, networked, and customized mold design navigation process, and when this mold design navigation system was placed in a web server, the designer could log in it via the internet and use the system for unified operation.

There are many algorithms regarding draft angle creation in previous literature; however, most of them use the internal parameter data of the model, which is feasible for CAD software development, but for general CAD software users, the data cannot be directly obtained due to limited CAD functions, thus, there remain difficulties in creation. On the other hand, the merits and demerits of different feature recognition methods are compared in literature, where it is observed that the feature recognition methods can effectively increase product design and manufacturing efficiency; however, after the recognition result is obtained, the subsequent design work still depends on manual operation, and its integration is seldom studied. Therefore, this paper aims to automate the recognition of draft angle, and to integrate it with the mold design navigation process established by Jong et al. [22]. A surface requiring draft design on a model is obtained from product contour vectors, geometrical relationship, program operation, and judgment; afterwards, the draft angle is automatically generated by the built-in function of CAD and its secondary development capabilities, which not 
only increases design efficiency, but also prevents errors in mold design and manufacturing.

\section{Mold Design Navigating Process}

In response to the complex demands of the market, a three-tier architecture [23] has been applied to develop systems, including the mold design navigating system. Based on the project management and relational database, this system establishes a standard automatic design process. This study attempted to integrate the database with the knowledge management system based on the three-tier system, in order to transform design knowledge and technology into actual processes and functionalities to help designers develop molds more rapidly and share knowledge more easily.

\subsection{Navigating Process Architecture}

The design navigating platform proposed in this study was built using the Creo Parametric 2.0 CAD software, using its ability to support web browsers that enables users to develop and design within authorized enterprise networks. In addition, the three-tier architecture developed by Jong [22] was introduced, to develop the program via the Microsoft.NET Framework. International Information Services (IIS) was employed as the web server. The webpage development languages used were ASP.NET 4.0(C\#), Asynchronous JavaScript and XML (AJAX) asynchronous access technology at the server end and the HTML, CSS, JavaScript and built-in Application Programmer Interface (API) set of the Creo Parametric 2.0 CAD software, providing the logic judgment and data processing the system required. Microsoft SQL Server 2008 Express Relational Database Management System (RDBMS) was employed for data access and storage. Regarding the media used for data storage and access, the CAD files generated in the design process were stored in the web disks of the Intranet. This system enables designers to carry out navigating design within the scope of Intranet through the embedded browser of CAD software. Meanwhile, the executives and clients on the Internet can also log onto the system to monitor the project process and design results. With CAD widely used by mold development personnel as the core tool, this platform realizes the seamless integration of project management, design navigating and knowledge management.

The mold design navigating process is applied to connect the entire design process, save the design information of various stages in a database, and feedback information at the proper time. Meanwhile, using a feature-oriented design, the features obtained at the conceptual design stage can be reused in the detailed mold design stage. Then, by project control and collaborative capabilities, a small number of experienced engineers can be in charge of the important early stages, such as the conceptual design and project analysis, while engineers with less experience can collaborate at the detailed mold design stage. In this way, the employees' capabilities can be fully used and the product development process can be accelerated. Through the secondary development interface pro- 
vided by the Creo Parametric 2.0, the design navigating process is developed for engineers to directly operate the CAD software according to the process.

\subsection{Secondary Development}

The entire mold design process includes many tedious and repetitive operations, such as file input, size computation, interference checks and, graphic capture, as well as operations including the assembling, save file, regeneration of relevant models. Automatic processing through programming can eliminate many redundant operations. Taking geometric outline computation as an example, although CAD provides many corresponding tools, requires numerous mouse clicks and selections to get the results. Through the direct writing of measuring tools using Pro/Web. Link, it takes only one click and selection by the users to obtain the dimensions of the geometric shape. The system then automatically determines the proper size of the mold core-cavity. In this way, it can accelerate the product design process and reduce human errors.

The Pro/Web. Link guideline manual [24] can help users to determine the corresponding purpose of the functions. According to the Eval Outline method, the function requires the presentation of the spatial matrix of the model (pfc Transform3D) as well as the feature removal item types (pfcModelItemTypes). The computation of the model size requires the geometric shape only and the unnecessary coordinates, points and axes can be removed using pfcModelItemTypes. The EvalOutline method is classified into the category of the pfcSolid. Therefore, it should be determined by its equivalent relations and the pfcModel type should be identified. The relevance of the pfcBaseSession can thus be obtained. Using the parental relationship with pfcSession, the type of MpfcCOMGlobal necessary for the initial announcement can be obtained accordingly. Such hierarchical relational search and type declaration can realize the access capabilities of CAD functions.

\subsection{User-Defined Feature (UDF)}

The UDF is used to reproduce the feature groups of the same shape, where some common features in product design are packaged as a UDF file, and this file is packaged with the required references and parameters for design in order to shorten design time. Figure 1(a) shows the creation process of the conventional Boss, where the Extrude function of CAD software is used, the sketch plane and direction reference are selected, and the sketch mode is entered. The boss body is extruded after the $2 \mathrm{D}$ section is drawn, and then the reinforcing ribs are designed. If UDF is used, the boss body and reinforcing rib features can be packaged as a UDF file. The boss is created provided its file is called from the UDF feature library, and relevant references and dimensions are given for rapid creation, as shown in Figure 1(b). This study uses the Pro/Web. Link to launch the UDF to create the automatic feature generation function, and the target feature can be rapidly created by providing the conditions and values of geometric references. 


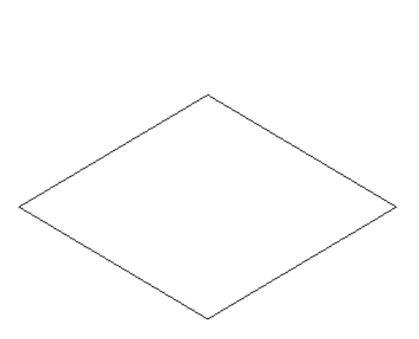

Select

Sketch Plane

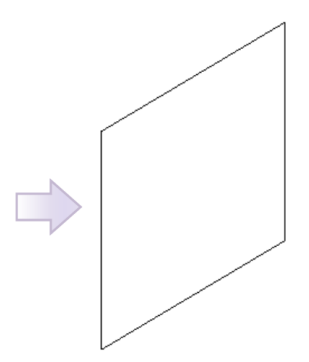

Select Sketch Orientation

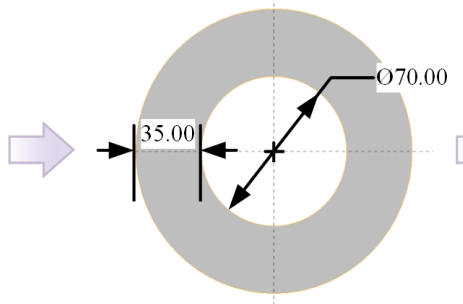

Create 2D Sketch

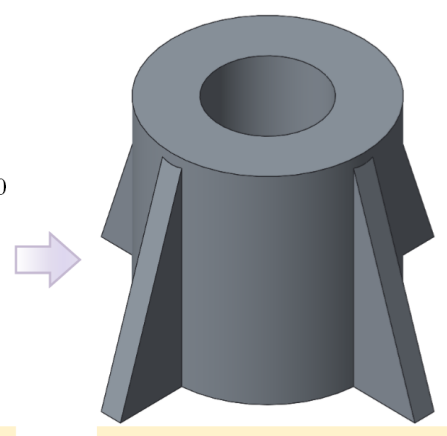

Extrude \& Add Ribs

(a) Conventional Feature Creation

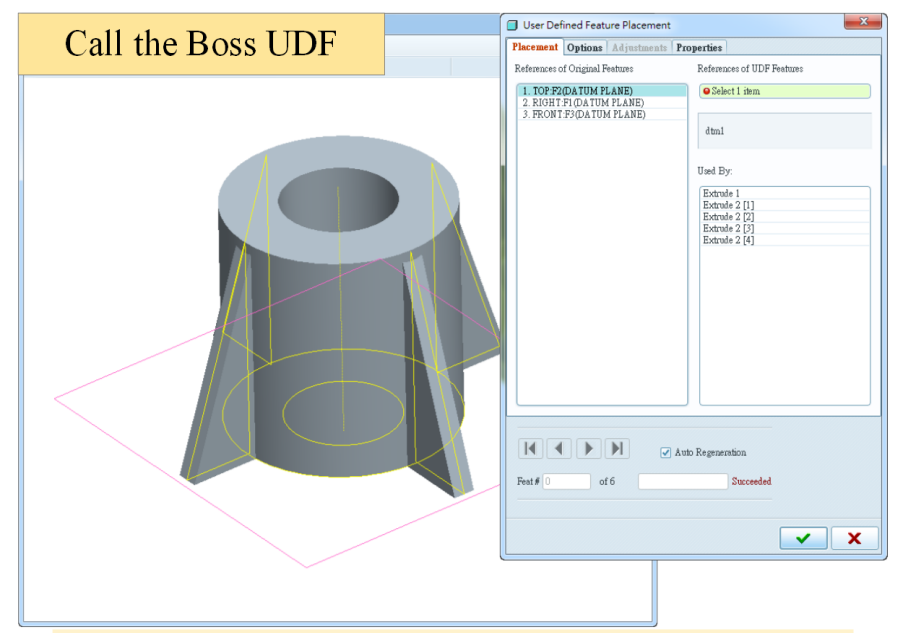

Select Placement References \& Modify Dimension

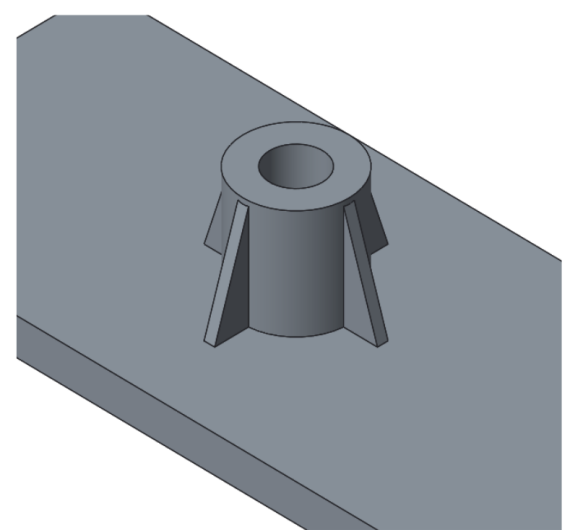

Add Boss on Target

(b) Feature Creation Using UDF

Figure 1. Boss design comparison without and with UDF.

\section{Draft-Angle Recognition and Creation}

In product design, due to the requirements of shape or any inappropriate molding consideration, a surface orthogonal to the parting surface may be created on the product, and such a completely normal surface has anbad effect on product demolding. A completely normal surface contacts the mold longer in the demolding process than a surface with a draft angle. As friction is longer, demolding is more difficult and is likely to damage the product surface, thus, influencing molding yield. Therefore, product draft angle must be considered in the mold concept design. The draft angle analysis and creation, as planned in this study, are built in the mold navigation process. In the initial concept phase of mold design, the secondary development language Pro/Web. Link is used in order to recognize a surface with insufficient draft angle according to the geometrical relationship among all the surfaces of demolding direction, and product model, where every surface to be drafted is classified, and the implemented draft function of CAD software is used for automatic draft angle creation. 


\subsection{Product Demolding Direction}

Product demolding direction must be determined before draft angle feature recognition in this study. In order to remove a product from the mold, the mold is divided into a Stationary Side and Moving Side. The Stationary Side is also called the Cavity Side, and the Moving Side is also called the Core Side. In this paper, the $\mathrm{Z}$-axis is used as the core/cavity side opening axis, $+\mathrm{Z}$ is the ejection direction, and $-\mathrm{Z}$ is the core demolding direction.

\subsection{Identification of Surfaces to Be Drafted}

The first step of draft angle analysis and creation is to determine the surfaces of the product to be added with a draft angle, which are filtered according to the two rules of this study.

\section{- Draft-rule-1}

In order to add a draft angle, the intersection of the to-be-drafted surface and $\mathrm{XZ}$ or YZ parallel plane must be a straight line. Besides, the included angle between the normal vector of any point on the surface and the $Z$-axis must be the same. In this paper, this rule is called the Straight Surface in the demolding direction (Z-axis). The three surfaces classified as a Straight Surface, which are Plane, Cylinder, and Tabulated Cylinder, can be found according to this rule. In the draft angle analysis of this paper, the three surface types are filtered out for further recognition in order to avoid spending time on surfaces that are impossible to draft.

\section{- Draft-rule-2}

When the filtered surface set is obtained by Rule1, the reference draft angle is specified to obtain the included angle between various surfaces of surface set and $\mathrm{Z}$-axis by geometric operation. If the included angle is smaller than the specified reference angle, the surface is classified into a surface set in order to add a draft angle. To check the included angle between various surfaces and the $\mathrm{Z}$-axis, the included angle equation $(\cos \theta=(\boldsymbol{A} \cdot \boldsymbol{B}) /\|A\|\|B\|)$ of trigonometric function is used to determine included angle $\theta$ between the normal vector of any point on each surface and the Z-axis, if $\theta<90^{\circ}$, the draft angle $d=90^{\circ}-\theta$, if $\theta>90^{\circ}$, then $d=\theta-90^{\circ}$. If $\mathrm{d}$ is smaller than the specified reference draft angle, it is classified into the set of surfaces to be drafted, otherwise, it is excluded from subsequent calculation. The set of surfaces required to add a draft angle can be converged by using the algorithms of Rule 1 and Rule 2.

\subsection{Classification of Surfaces to Be Drafted}

When the surfaces to be drafted are filtered, the draft positions will be grouped into the collected surface set in order to create the draft angle in the next stages.

\section{Step 1: Identification of top/bottom face of surface to be drafted}

First, based on the Z-axis, the heights of the edges of the surface to be drafted are compared in order to obtain the top and bottom margins of the surface to be drafted. When the top margin and bottom margin are obtained, the adjacent surfaces are taken as the top surface and bottom surface, and the $\mathrm{Z}$ components 
of the normal vectors of top and bottom surfaces are used for classification. The height of the two edges is checked, as follows:

- Face-rule-1

If the two endpoints of one of the two edges are higher than or equal to the peak point of the other edge, then this edge is higher. On the contrary, if the two endpoints of an edge on the $\mathrm{Z}$-axis are lower than or equal to the peak point of the other edge, then this edge is lower. As shown in Figure 2(a), the starting point $\mathrm{P}_{\mathrm{s} 2}$ and end point $\mathrm{P}_{\mathrm{e} 2}$ of Edge $\mathrm{E}_{2}$ are greater than or equal to the higher endpoint $\mathrm{P}_{\mathrm{e} 1}$ on Edge $\mathrm{E}_{1}$, then Edge $_{2}$ must be above Edge $\mathrm{e}_{1}$.

- Face-rule-2

If one of the two edges has only one endpoint higher than or equal to the peak point of the other edge, and the edge of the upper edge cannot be completely determined. Then, the slopes of the two edges should be judged, the smaller absolute value of the slope is taken as the upper edge, and the lower edge is deduced by this rule, as shown in Figure 2(b). The starting point $\mathrm{P}_{\mathrm{s} 2}$ of $\mathrm{Edge}_{2}$ is greater than or equal to the higher endpoint $\mathrm{P}_{\mathrm{e} 1}$ on Edge $\mathrm{E}_{1}$, but $\mathrm{P}_{\mathrm{e} 2}$ is smaller than $\mathrm{P}_{\mathrm{e} 1}$, the upper one cannot be determined. In this case, this study compares the slopes of the two edges, and the slope of Edge $e_{2}$ is smaller than that of Edge $e_{1}$, thus, Edge $_{2}$ is above Edge ${ }_{1}$. All the edges on the surface are matched by circulation in order to obtain the adjacent planes corresponding to the top and bottom margins, i.e. top surface and bottom surface.

\section{Step 2: Identification of to-be-drafted surfaces}

When the top and bottom surfaces of a surface to be drafted are obtained using the aforesaid method, the normal vector $\mathrm{Z}$-axis components are checked, and the three major types, as shown in Figure 3, are obtained. Type I: two Z components are in the same direction, as shown in Figure 3(a), and this type is usually a core/cavity side convex feature, such as a rib or boss hole. Type II: two $\mathrm{Z}$ components are in opposite directions and far from each other, as shown in Figure $3(\mathrm{~b})$, and this type may be a hole or product side. If the top and bottom margins are on one side of the top and bottom internal contours, respectively, it
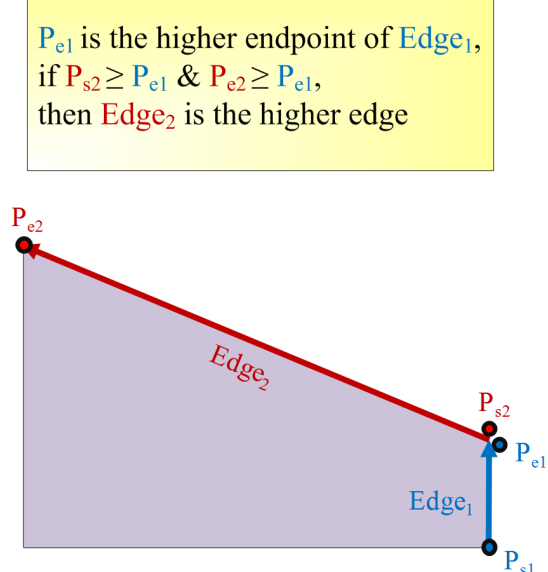

(a)
$P_{\mathrm{el}}$ is the higher endpoint of Edge $_{1}$, if $\mathrm{P}_{\mathrm{s} 2} \geq \mathrm{P}_{\mathrm{e} 1}$ \& $\mathrm{P}_{\mathrm{e} 2}<\mathrm{P}_{\mathrm{e} 1}$, and slope of Edge $\mathrm{E}_{2}<$ slope of Edge $\mathrm{E}_{1}$, then $\mathrm{Edge}_{2}$ is the higher edge

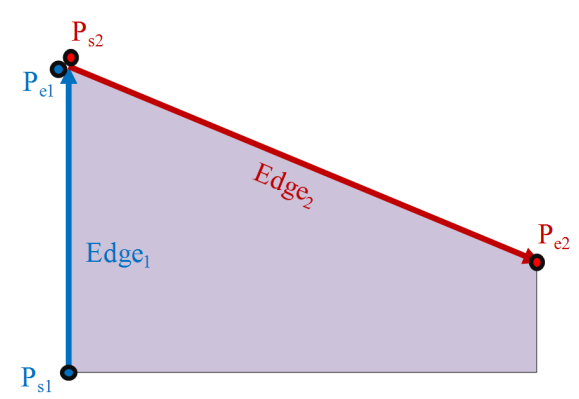

(b)

Figure 2. Judgment of edge height of surface to be drafted. 


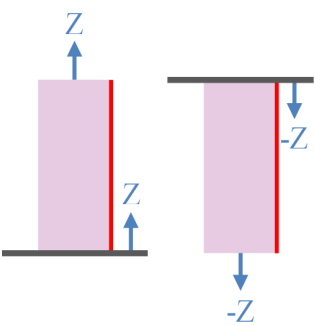

Core/Cavity Side

(a)

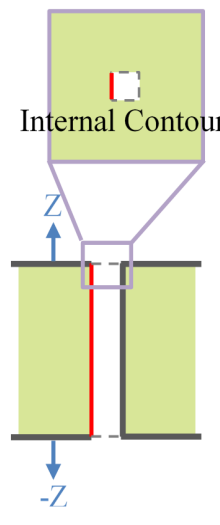

Hole

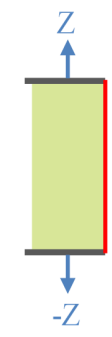

(b)

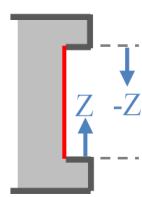

Undercut

(c)

Figure 3. Classification of positions of surfaces to be drafted.

is identified as a hole type, otherwise it is classified as a side type. Type III: two Z components are in opposite directions and facing each other, as shown in Figure $3(\mathrm{c})$, and this type is classified as an undercut of a product.

When the positions of surfaces to be drafted are obtained, different draft angle creation directions can be set according to the positions. Type I: the surface of the core/cavity side must be drafted according to its orientation. Type II: on the surface to be drafted of a hole or product side, the draft direction is defined according to design requirements. Type III: for the undercut, an additional mold mechanism should be designed for demolding, such as a slider or lifter.

\subsection{Automatic Draft Angle Creation}

When all the surfaces of a product to be drafted are identified by the secondary development language Pro/Web. Link, the UDF function of Pro/E is used to create the draft angle on the surface, and the automatic draft function is implemented for the first type of surface to be drafted, as concluded in the previous section, i.e. the surface to be drafted is determined on the core/cavity side. Therefore, only the set of the first type of surfaces to be drafted is automated, because among the three types, only the first type can obtain the draft direction according to the geometric position. In terms of Type II, the designer should set the draft direction according to requirements. In terms of Type III, the undercut position depends on the mold mechanism. Therefore, this study only searches and recognizes the second and the third types of draft surface sets without making any further automatic draft.

\subsubsection{Selection of Draft Hinge}

In Pro/E, the draft angle is created by using the surface of the product to be drafted, as well as a hinge or edge to rotate the draft surface, in order to generate the draft angle feature. This study will use a hinge to create the draft angle, which is set on the top or bottom surface of the surface to be drafted according to the draft hinge, and divided into thickness cutting draft or thickness adding draft, as shown in Figure 4. Generally, in mold industry engineers inclines to 


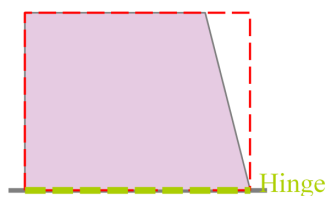

(b) Create Draft Angle by Cutting Thickness

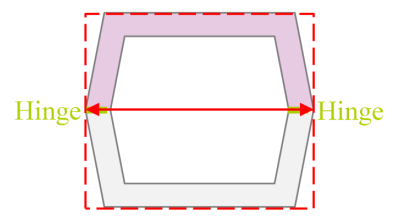

(c) Create Draft Angle by Cutting Thickness

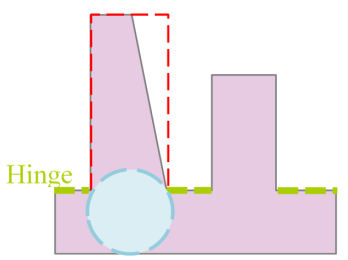

(e) Create Draft Angle by Cutting Thickness

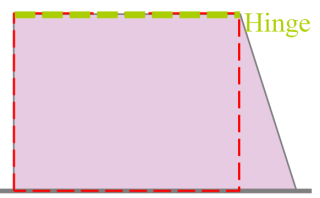

(a) Create Draft Angle by Adding Thickness

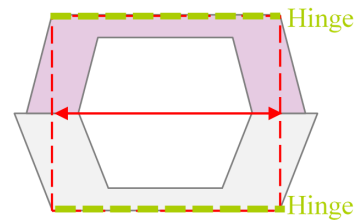

(d) Create Draft Angle by Adding Thickness

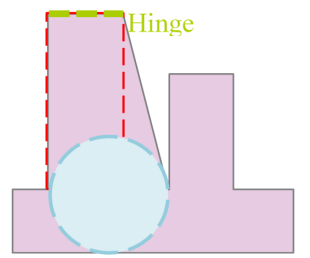

(f) Create Draft Angle by Adding Thickness

Figure 4. Draft hinge comparison.

use the thickness cutting draft, and its advantages include 1) the maximum overall dimension of the product can be controlled, and failure in assembly of matching parts can be avoided. Figure 4(c) shows that the core and cavity sides are thickness cutting draft, and it is observed that in this draft mode, the maximum size of product can be fixed, and assembly remains normal when the core and cavity draft angles are different. Figure 4(d) shows the thickness adding draft, where the maximum size of the product is changed, and assembly fails when the core and cavity draft angles are different. 2) Interference between the mechanisms inside the product can be avoided, and the average thickness is controlled to avoid shrinkage defects. Figure 4(e) shows that the mechanism inside the product is drafted by cutting thickness. Figure 4(f) shows that the mechanism inside the product is drafted by adding thickness. It is observed in the two figures that, using added thickness for a product mechanism draft may result in excessive mechanism root thickness, which is likely to cause a shrinking mark; and if the distance between the two mechanisms is small, it is likely to cause interference between the mechanisms. Therefore, this study will use the thickness cutting draft for automatic draft angle.

In addition, the selection of a draft hinge may influence the geometric shape of the drafted product, as shown in Figure 5. Figure 5(a) shows the curved hinge. It is observed that, after a curved hinge is used for a draft, the product shape changes from a straight line into a curve, because when the surface is used as the draft hinge, and the draft feature is sectioned along the hinge, the hinge positions of the section are different. Therefore, when the draft angle is fixed, the hinges at different depths have different thickness cutting effects on the product. 


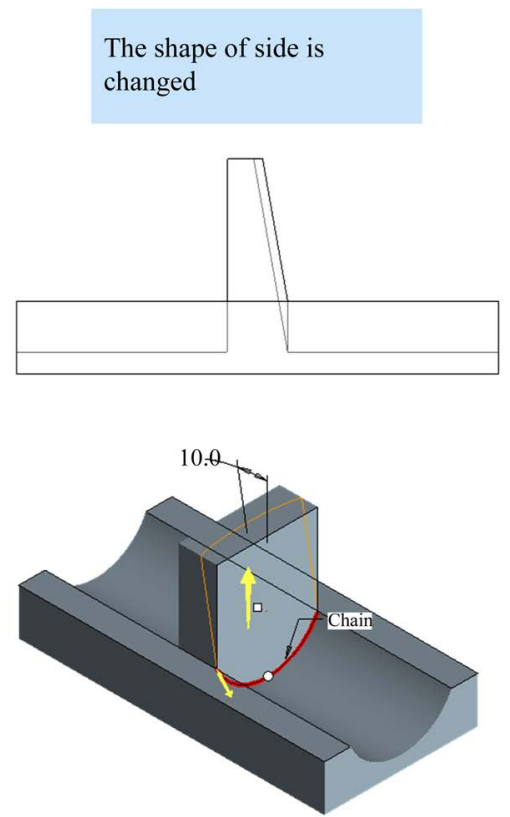

(a) Draft by Non-Flat Hinge

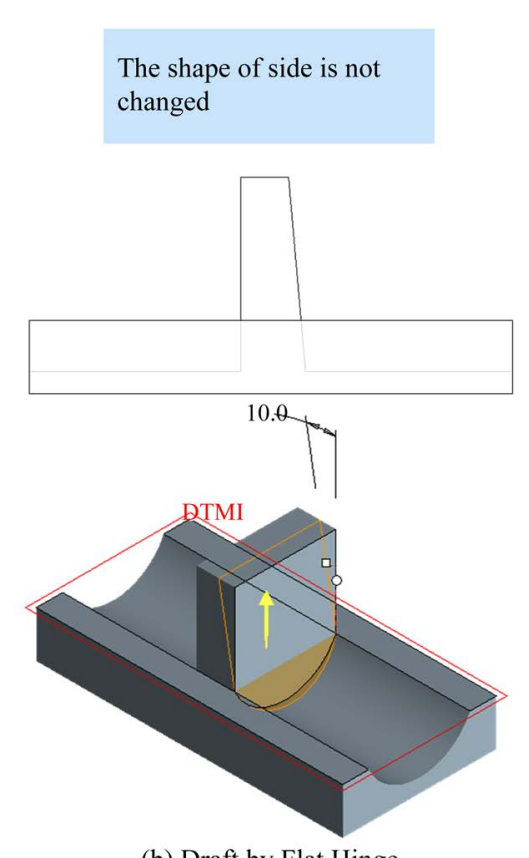

(b) Draft by Flat Hinge

Figure 5. Non-flat draft hinge comparison.

It is observed that, at the same angle, the hinge in the deeper position has greater thickness cutting effect on the product, as such the original shape of the model surface cannot be maintained. However, the modification that changes appearance is seldom accepted by the product designers. Therefore, in this case, this study creates a flat reference plane as the draft hinge, as shown in Figure 5(b), where the original external dimension of the product is maintained after the draft angle is created.

In the Pro/E environment, the draft angle creation function is performed by the designer defining the draft surface, draft hinge, draft direction, and draft angle; in other words, there are four steps, including clicking three reference objects and entering one value. This study uses secondary development language Pro/Web. Link, the UDF function, and the draft surface recognition rules of this paper to automatically capture and import the reference objects into the draft UDF, meaning the designer does not have to manually decide the draft surface, draft hinge, or draft direction, thus, the draft-angle operating procedure is effectively shortened.

\subsubsection{Constraints of Automatic Draft}

In the automatic draft function, as designed in this study, when the proximal face of the top or bottom margin of the surface to be drafted is a fillet surface, the draft angle feature cannot be directly added. When cutting thickness or adding thickness is used, the fillet feature surface cannot be maintained. Thus, the draft angle cannot be directly created, as the fillet feature surface must be removed first, and the fillet surface is rebuilt for the top margin after the draft is completed. Therefore, though the automatic draft angle creation function of this study cannot be directly used, recognition is still quite useful. The types of geo- 
metric surfaces are defined by the secondary development language Pro/Web. Link, and there is a coding rule for recognition. Therefore, this study uses this characteristic to conclude the combination of surfaces to be drafted with a fillet, as shown in Table 1 , in order to recognize the surface to be drafted with a fillet.

\section{Case Study}

This study uses a mobile phone shell and front frame of LCD for draft angle analysis and automatic draft angle creation. Moreover, the Pro/Web. Link and macro (Mapkey) function are used for color marking. Compared with the built-in function of Pro/E, the advantage of analysis result rendering in this study is that a geometric surface with an insufficient draft angle is marked, and the coloring effect will keep showing in the analytical model, thus, contributing to draft angle design.

In this case, the top case of a flip phone is used for draft analysis. The target draft angle is set as $3^{\circ}$, and the built-in draft analysis result of Pro/E is as shown in Figure 6(a). Figure 6(b) shows the analysis result of this study. It is observed

Table 1. Coded combination of surfaces to be drafted with a fillet.

\begin{tabular}{ccc}
\hline Pro/Web. Link Surface Type & $\begin{array}{c}\text { Pro/Web. Link } \\
\text { Surface Type Code }\end{array}$ & $\begin{array}{c}\text { Code of Surface } \\
\text { with Round }\end{array}$ \\
\hline SURFACE_PLANE & 0 & 0 next to 1 \\
SURFACE_CYLINDER & 1 & 1 next to 3 \\
SURFACE_CONE & 2 & - \\
SURFACE_TORUS & 3 & - \\
SURFACE_RULED & 4 & - \\
SURFACE_REVOLVED & 5 & 6 next to 7 \\
SURFACE_TABULATED_CYLINDER & 6 & - \\
SURFACE_FILLET & 7 & - \\
\hline
\end{tabular}

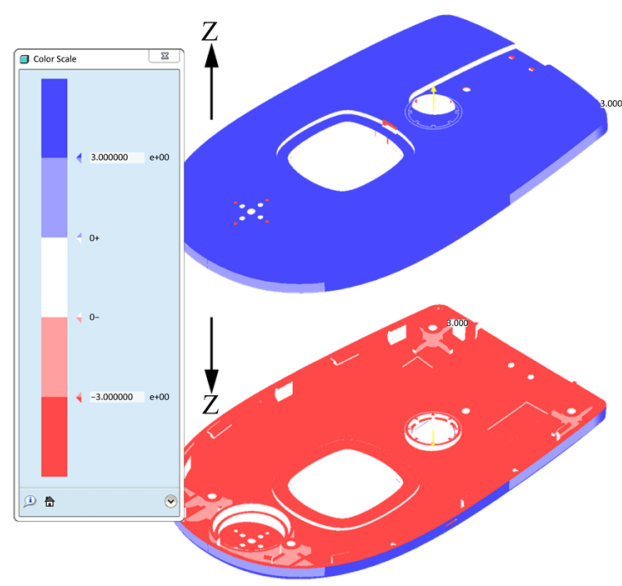

(a) Draft Analysis in Pro/E

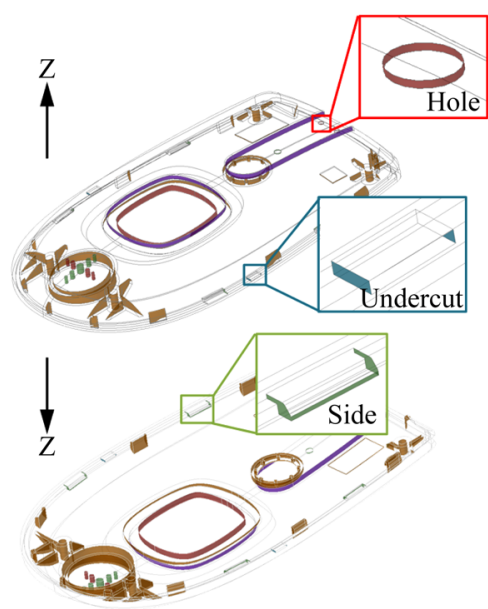

(b) Draft Analysis in This Study

Figure 6. Draft angle analysis result. 
that, purple is the cavity $(+Z)$ side surface to be drafted, orange is the core $(-Z)$ side surface to be drafted, red is the hole, blue is the undercut, and green is the surface to be drafted of the geometric edge of the model. There is misrecognition in this case, as shown in Figure 7. The green part should be the hole geometry; however, as the top margin consists of two surfaces, it results in misrecognition during the operation rule of this study. Finally, the quantity statistics are as shown in Table 2. The total quantity of surfaces to be drafted is 180 , the surfaces available for automatic draft angle creation in this study are the core and cavity side surfaces, and is without an adjacent fillet. It is observed that there are 121 surfaces. The automatic draft angle creation is implemented for 121 surfaces, where six of them can directly use the root plane as a draft hinge, each surface can be built by clicking only once using the automatic draft angle creation function, and the number of clicks of manual operation is as shown in Table 3.

The main difference between the aforesaid case is that the traditional manual design must use the CAD software function, and the references are clicked step by step according to the interface requirement in order to complete the creation

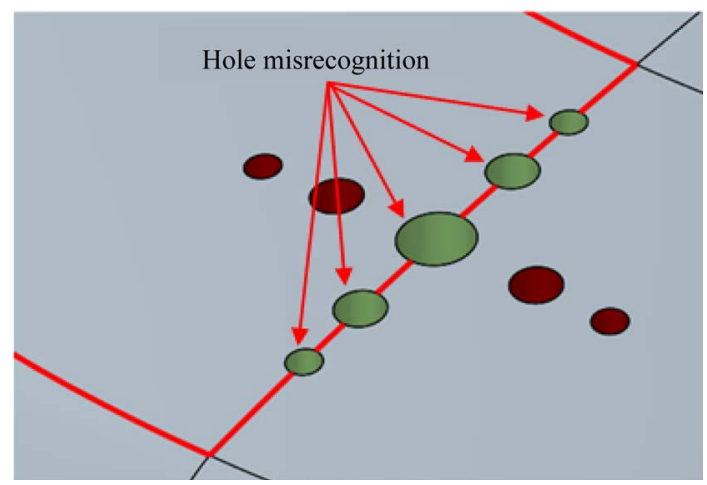

Figure 7. Hole misrecognition.

Table 2. Coded combination of surfaces to be drafted with a fillet.

\begin{tabular}{cccc}
\hline Position Type & Total Number & Without Round & With Round \\
\hline Cavity side surfaces & 6 & 0 & 6 \\
Core side surfaces & 129 & 121 & 8 \\
Hole surfaces & 16 & 12 & 4 \\
Side surfaces & 25 & 21 & 4 \\
Undercut surfaces & 4 & 4 & 0 \\
Total & 180 & 158 & 22 \\
\hline
\end{tabular}

Table 3. Comparison of number of clicks.

\begin{tabular}{ccc}
\hline & Traditional & Automatic \\
\hline Draft by root surface hinge (6 surfaces) & 42 & 6 \\
Draft by datum hinge (115 surfaces) & 1150 & 115 \\
Total clicks & 1192 & 121 \\
\hline
\end{tabular}


of features; whereas, the algorithm and automation developed using Pro/Web. Link in this study can help engineers to rapidly make decisions on design, and the UDF function can rapidly create draft features to complete the design, thus, increasing the engineers' design efficiency.

\section{Conclusion}

This study uses the geometrical relationships among product surfaces, points, and curves to develop an algorithm, such that the product draft angle is recognized with the specified angle, and the automation function improves the accuracy and time of engineers in draft design. Feature recognition algorithm can automatically identify $90 \%$ of the surface to be drafted, so that would reduce $80 \%$ of the mouse clicks during the design process. This study sum up the three types of surfaces to be drafted so it simplifies the complex model and converges the items need to be classify. Therefore the correction of identification is improved. The surface to be drafted can be automatic colored, so the engineers will knew the proper way to design the mechanism. Since the filet near the curve surface cannot create the features to be drafted automatically in CAD software, it is more efficient to do the drafted design before the fillet design. In this study, there are three types of methods to create the drafted mold feature are established and has already applied first type of method. In the future, second and third types of method will be accomplished by taking parting line and surface into consideration in the algorithm. In future research we will focus on methods of distinguish discontinuous surfaces, and take the curved surface area and height into account, so that the algorithm of drafting feature is more complete and improve the function.

\section{References}

[1] Ulrich, T.K. and Eppinger, D.S. (2004) Product Design and Development, McGrawHill Inc., New York.

[2] Tokuyama, Y. and Bae, S. (1999) An Approximate Method for Generating Draft on a Free-Form Surface. The Visual Computer, 15, 1-8.

https://doi.org/10.1007/s003710050158

[3] Yan, Y. and Tan, S.T. (2004) Adding Draft Angles on Mechanical Components Containing Constant Radius Blending Surfaces. Computer-Aided Design, 36, 565580. https://doi.org/10.1016/S0010-4485(03)00135-0

[4] Schreve, K. (2009) Sufficient Conditions for Draft Angles on General B-Spline Surface. Computer-Aided Design, 41, 681-684. https://doi.org/10.1016/j.cad.2008.12.007

[5] Zhou, P. and Qian, W.H. (2012) Adding Draft Angle to B-Spline and NURBS Surfaces. Computer-Aided Design, 44, 617-625.

https://doi.org/10.1016/j.cad.2012.02.010

[6] Nee, A.Y.C., Fu, M.W., Fuh, J.Y.H., Lee, K.S. and Zhang, Y.F. (1998) Automatic Determination of 3-D Parting Lines and Surfaces in Plastic Injection Mould Design. CIRP Annals-Manufacturing Technology, 47, 95-98. https://doi.org/10.1016/S0007-8506(07)62793-0

[7] Fu, M.W., Nee, A.Y.C. and Fuh, J.Y.H. (2002) The Application of Surface Visibility 
and Moldability to Parting Line Generation. Computer-Aided Design, 34, 469-480. https://doi.org/10.1016/S0010-4485(01)00117-8

[8] Weinstein, M. and Manoochehri, S. (1997) Optimum Parting Line Design of Molded and Cast Parts for Manufacturability. Journal of Manufacturing Systems, 16, 1-12. https://doi.org/10.1016/S0278-6125(97)88401-4

[9] Mercado-Colmenero, J.M., Paramio, M.A.R., Perez-Garcia, J.M. and MartinDoñate, C. (2016) A New Hybrid Method for Demoldability Analysis of Discrete Geometries. Computer-Aided Design, 80, 43-60. https://doi.org/10.1016/j.cad.2016.06.006

[10] Singh, R., Madan, J. and Kumar, R. (2014) Automated Identification of Complex Undercut Features for Side-Core Design for Die-Casting Parts. Proceedings of the Institution of Mechanical Engineers, Part B: Journal of Engineering Manufacture, 228, 1138-1152. https://doi.org/10.1177/0954405413514744

[11] Vandenbrande, J.H. and Requicha, A.A.G. (1993) Spatial Reasoning for the Automatic Recognition of Machinable Features in Solid Models. IEEE Transactions Pattern Analysis and Machine Intelligence, 15, 1269-1285. https://doi.org/10.1109/34.250845

[12] Vandenbrande, J.H. and Requicha, A.A.G. (1994) Geometric Computation for the Recognition of Spatially Interacting Machinable Features. Advances in Feature Based Manufacturing, Elsevier Science, 20, 83-106. https://doi.org/10.1016/B978-0-444-81600-9.50010-9

[13] Verma, A.K. and Rajotia, S. (2007) A Hint-Based Machining Feature Recognition System for 2.5 D Parts. International Journal of Production Research, 46, 1515-1537. https://doi.org/10.1080/00207540600919373

[14] Babic, B., Nesic, N. and Miljkovic, Z. (2008) A Review of Automated Feature Recognition with Rule-Based Pattern Recognition. Computers in Industry, 59, 321-337. https://doi.org/10.1016/j.compind.2007.09.001

[15] Li, W.D., Ong, S.K. and Nee, A.Y.C. (2010) A Hybrid Method for Recognizing Interacting Machining Features. International Journal of Production Research, 41, 1887-1908. https://doi.org/10.1080/0020754031000123868

[16] Lai, J.Y., Wang, M.H., You, Z.W., Chiu, Y.K., Hsu, C.H., Tsai, Y.C. and Huang, C.Y. (2016) Recognition of Virtual Loops on 3D CAD Models Based on the B-Rep Model. Engineering with Computers, 32, 593-606. https://doi.org/10.1007/s00366-016-0436-3

[17] Turng, L.S., DeAugistine, D. and Taam, B. (1998) A Knowledge Base System for the Design and Manufacture of Injection-Molded Plastic Products. IEEE Information Technology Conference, Syracuse, 3 September 1998, 95-98. https://doi.org/10.1109/it.1998.713390

[18] Fuh, J.Y.H., Zhang, Y.F., Nee, A.Y.C. and Fu, M.W. (2004) Computer-Aided Injection Mold Design and Manufacture. Marcel Dekker, New York.

[19] Chan, W.M., Yan, L., Xiang, W. and Cheok, B.T. (2003) A 3D CAD Knowledge-Based Assisted Injection Mould Design System. International Journal of Advanced Manufacturing Technology, 22, 387-395.

https://doi.org/10.1007/s00170-002-1514-9

[20] Jong, W.R., Wu, C.H., Liu, H.H. and Li, M.Y. (2009) A Collaborative Navigation System for Concurrent Mold Design. International Journal of Advanced Manufacturing Technology, 40, 215-225. https://doi.org/10.1007/s00170-007-1328-x

[21] Jong, W.R., Wu, C.H. and Li, M.Y. (2011) Feature-Based Integration of Conceptual and Detailed Mould Design. International Journal of Production Research, 49, 4833-4855. https://doi.org/10.1080/00207543.2010.504421 
[22] Jong, W.R., Wu, C.H. and Li, M.Y. (2011) Web-Based Navigating System for Conceptual Mold Design with Knowledge Management. International Journal of Production Research, 49, 553-567. https://doi.org/10.1080/00207540903439830

[23] Eckerson, W.W. (1995) Three Tier Client/Server Architectures: Achieving Scalability, Performance, and Efficiency in Client Server Applications. Open Information Systems, 3, 46-50.

[24] Parametric Technology Corporation (2009) Pro/ENGINEER Wildfire 5.0 Pro/ Web.Link User's Guide. PTC, Needham.

Submit or recommend next manuscript to SCIRP and we will provide best service for you:

Accepting pre-submission inquiries through Email, Facebook, LinkedIn, Twitter, etc. A wide selection of journals (inclusive of 9 subjects, more than 200 journals)

Providing 24-hour high-quality service

User-friendly online submission system

Fair and swift peer-review system

Efficient typesetting and proofreading procedure

Display of the result of downloads and visits, as well as the number of cited articles

Maximum dissemination of your research work

Submit your manuscript at: http://papersubmission.scirp.org/

Or contact jsea@scirp.org 